\title{
Human norovirus transmission and evolution in a changing world
}

\author{
Miranda de Graaf, Janko van Beek and Marion P. G. Koopmans
}

Abstract | Norovirus infections are a major cause of gastroenteritis, and outbreaks occur frequently. Several factors are currently increasing the challenge posed by norovirus infections to global health, notably the increasing number of infections in immunocompromised individuals, who are more susceptible to disease, and the globalization of the food industry, which enables large norovirus outbreaks to occur on an international scale. Furthermore, the rapid rate of the genetic and antigenic evolution of circulating noroviruses complicates the development of vaccines and therapies that are required to counter these challenges. In this Review, we describe recent advances in the study of the transmission, pathogenesis and evolution of human noroviruses, and consider the ongoing risk of norovirus outbreaks, together with the future prospects for therapeutics, in a rapidly changing world.

\section{Genogroups} Groups of related viruses within a genus.

\section{Polyprotein}

A large protein that is cleaved into separate smaller proteins with different biological functions.
Department of Viroscience, Erasmus University Medical Center, P.O. Box 2040 . 3000CA Rotterdam, The Netherlands. Correspondence to M.P.G.K. m.koopmans@erasmusmc.nl
The majority of all non-bacterial gastroenteritis outbreaks are caused by human noroviruses ${ }^{1}$. These viruses are highly infectious, as even a few particles can cause disease, and infected individuals shed high loads of virus ${ }^{2,3}$. Transmission occurs by the faecal-oral route, either through contact with infected individuals or through exposure to contaminated food and water or to infectious aerosols that are produced by vomiting ${ }^{4-7}$. As a result of this high infectivity and efficient transmission, newly emerged strains of norovirus can cause global epidemics ${ }^{8}$.

Norovirus infections are self-limiting in healthy individuals but are associated with severe complications in immunocompromised individuals, the elderly and young children ${ }^{9-13}$ (BOX 1). Noroviruses have not always been associated with more severe disease in individuals with impaired immune functions, and this recent pattern is thought to be linked to genetic differences between emerging and historical human strains of norovirus. Studies to characterize these differences have until recently been hampered by the uncultivability of noroviruses, but a cell culture model has now been described for human noroviruses, and ongoing studies may provide molecular insights into the increased risk posed by emerging strains of norovirus ${ }^{14}$.

The genus Norovirus belongs to the family Caliciviridae and contains only a single species, Norwalk virus; this species is divided into at least six genogroups, which are further subdivided into at least 30 genotypes $^{15}$. Viruses from genogroups GI, GII and GIV are known to infect humans. In the past decade, viruses of the genotype GII.4 have caused the majority of clinical cases $^{8,15,16}$, although the newly emerged GII.P17-GII.17 genotype has recently become the predominant strain in some parts of Asia ${ }^{17}$.

Human noroviruses have a non-segmented positivestrand RNA genome, of approximately $7.5 \mathrm{~kb}$, that contains three ORFs (FIG. 1). These ORFs encode a large non-structural polyprotein (ORF1), the major structural protein VP1 (ORF2) and the minor structural protein VP2 (ORF3) ${ }^{18}$. The icosahedral viral particle capsid is composed of 90 dimers of VP1, which consists of a shell (S) domain and a protruding $(\mathrm{P})$ domain. The $\mathrm{P}$ domain is responsible for binding to histo-blood group antigens (HBGAs), which function as receptors or co-receptors on host cells ${ }^{19}$, and it contains important determinants of antigenicity ${ }^{19}$. Viral particles contain only a few copies of VP2, which are associated with the interior surface of the capsid formed by the $\mathrm{S}$ domain of VP1 (REFS 19,20). Currently, there is no licensed norovirus vaccine available, but most vaccine strategies are focused on VP1 (REF. 21).

The mechanism of internalization of human noroviruses after binding to HBGAs is not known but may be dependent on dynamin and cholesterol, as has been shown for murine noroviruses ${ }^{18,22}$. Replication of the virus occurs in the cytoplasm in close association with host-derived membrane complexes (FIG. 1; comprehensively reviewed in REFS 18,22). The mechanisms of assembly and release of human noroviruses remain largely unknown.

The increasing evidence for the risk that is posed by noroviruses to global health calls for a better understanding of these molecular aspects of norovirus pathogenesis 


\section{Box 1 | The social cost of norovirus infections}

Norovirus infection is associated with $18 \%$ of all cases of gastroenteritis worldwide ${ }^{28}$, but of most concern in the clinic is the severe disease that can occur in individuals who have impaired immunity. In healthy adults, the infection is self-limiting, and clinical symptoms tend to last for 2-3 days ${ }^{2,29}$. By contrast, in the elderly, young children and individuals who have impaired immune functions, clinical symptoms can be much more severe and last longer ${ }^{10,12,13}$. Peaks in mortality in the elderly have been associated with seasonal peaks in norovirus infections, although noroviruses are rarely noted as the cause of death on death certificates, and definitive proof of causality remains to be determined $^{10}$. The most frequently reported causes of death that are associated with norovirus infection are aspiration or pneumonia, but other causes of death include gastroenteritis, sepsis, cardiac complications, necrotizing enterocolitis, malnutrition, acute gastrointestinal bleeding and colon perforation ${ }^{11}$. In resource-limited countries, gastroenteritis is an important cause of morbidity and mortality; however, owing to a lack of molecular diagnostic tools in these countries, it is difficult to assess which cases of gastroenteritis are associated with norovirus infections ${ }^{110}$. In mice, malnutrition is associated with more severe norovirus infections, as defined by weight loss, impaired control of infection, reduced antiviral-antibody responses, enhanced viral evolution and loss of protective immunity ${ }^{111}$, but it remains to be seen whether this also applies to norovirus infections in malnourished children.

Patients who have underlying impaired immunity can become chronically infected with noroviruses for prolonged periods of time, in some cases for more than a year ${ }^{12}$. Chronic norovirus infections can be devastating in patients who are already frail, affecting their quality of life and their recovery. Prolonged norovirus infections have been reported in individuals of all ages with congenital immunodeficiencies, in transplant patients who are receiving immunosuppressive therapy, in patients undergoing cancer chemotherapy and in individuals who are infected with HIV ${ }^{12,99}$. Owing to the increasing number of patients receiving solid organ or haematopoietic stem cell transplants, this risk group has expanded substantially in the past few decades. Noroviruses are frequently detected in younger children with inherited immune deficiencies, and these infections can last for more than 9.5 months ${ }^{112}$. A chronic norovirus infection in an immunocompromised individual arises because the individual is unable to clear the virus following acute gastroenteritis. By contrast, strains of human norovirus that are able to cause persistent infections in otherwise healthy individuals have not been described to date.

Nosocomial transmission

The transmission of an

infectious disease in a hospital

setting

Herd immunity

General immunity of a host population to a pathogen,

mediated by immunity

acquired by a high proportion

of individuals within the

population.

Gnotobiotic

Pertaining to an animal: germ-free, or having known

associated microorganisms. and evolution, together with an improved characterization of the genetic determinants of transmission and disease severity. In this Review, we describe recent advances in the study of norovirus transmission and pathogenesis, with an emphasis on the role of viral evolution and genetic diversity. Furthermore, we highlight the aspects of norovirus biology for which data are most lacking and the new approaches to modelling norovirus infection in the laboratory, such as cell culture systems and smallanimal models, that may be used to study them. Finally, we discuss the current state of the development of treatment strategies and vaccines.

\section{Transmission}

Surveillance through national and international collaborative networks, such as CaliciNet and NoroNet, has provided important insights into how different strains of human norovirus correspond to modes of transmission and outbreak settings. Strains of the GII.4 genotype caused $70-80 \%$ of all reported outbreaks over the past 13 years or $\mathrm{so}^{23}$, but the prevalence of infecting genotypes differs between human populations and routes of transmission ${ }^{16}$. Genotype GII.4 is more often associated with transmission mediated by person-to-person contact than with other types of transmission, whereas non-GII.4 genotypes, such as GI.3, GI.6, GI.7, GII.3,
GII.6 and GII.12, are more often associated with foodborne transmission ${ }^{5}$. Genogroup GI strains are more often associated with waterborne transmission than GII strains 7 , a trait that may relate to the proposal that GI strains have a higher stability in water than GII strains. As strains may adapt to host factors that vary according to the population that is infected, such as age, health and pre-existing immunity, differences in the epidemiology of norovirus genotypes in community settings are likely to influence the evolution of the genotypes (FIC. 2).

Foodborne transmission is an important route for the global spread of noroviruses ${ }^{5}$ and can occur either when food handlers contaminate food on site or during the earlier steps of food production ${ }^{24}$. For example, shellfish that are cultivated in coastal areas can be contaminated by faecal discharge ${ }^{25}$, and products such as fresh and frozen berries can be contaminated by irrigation with sewage-contaminated water or by contact with infected personnel during harvesting and processing. Foodborne outbreak events occur frequently and are a potential source of transmission of strains between different parts of the world, given the globalization of the food chain. These outbreaks can include mixtures of norovirus strains ${ }^{7}$, thus increasing the risk of viral recombination. The global scale of foodborne outbreaks of noroviruses can be difficult to recognize because the epidemiology of outbreaks is often tracked independently by individual countries; nonetheless, retrospective studies have shown that approximately $7 \%$ of foodborne outbreaks of noroviruses are part of an international event with a common source ${ }^{26}$. Globally, noroviruses rank among the top causes of foodborne disease ${ }^{27}$.

Nosocomial transmission of noroviruses in hospitals is a major burden for in-patient services ${ }^{28}$. Individuals may shed norovirus particles in considerable numbers for several weeks after the resolution of symptoms ${ }^{29}$, possibly acting as a source for nosocomial transmission ${ }^{30}$. However, analyses of nosocomial outbreaks suggest that most of these outbreaks are the result of transmission from symptomatic shedders ${ }^{30}$. In a hospital setting, immunocompromised patients who are chronically infected with a norovirus and are symptomatic can act as a reservoir of the virus and may contribute to nosocomial transmission ${ }^{31,32}$. As a consequence of prolonged shedding and limited immune pressure, these immunocompromised patients can harbour numerous norovirus variants. The intrahost viral variation in a chronic shedder can mimic the antigenic variations that are seen between consecutive human norovirus pandemics, and some of these variants may be able to escape herd immunity ${ }^{33}$.

Infections of humans with animal norovirus strains have not yet been reported, but there is some evidence for the transmission of noroviruses between different host species. Human noroviruses have been detected in the stool of pigs, cattle and $\operatorname{dog} s^{34,35}$, and gnotobiotic calves and pigs can become experimentally infected with human GII.4 strains ${ }^{36,37}$. Furthermore, canine seroprevalence to different human norovirus genotypes resembles the seroprevalence in the human population ${ }^{38}$, and serum antibodies against bovine and canine noroviruses 


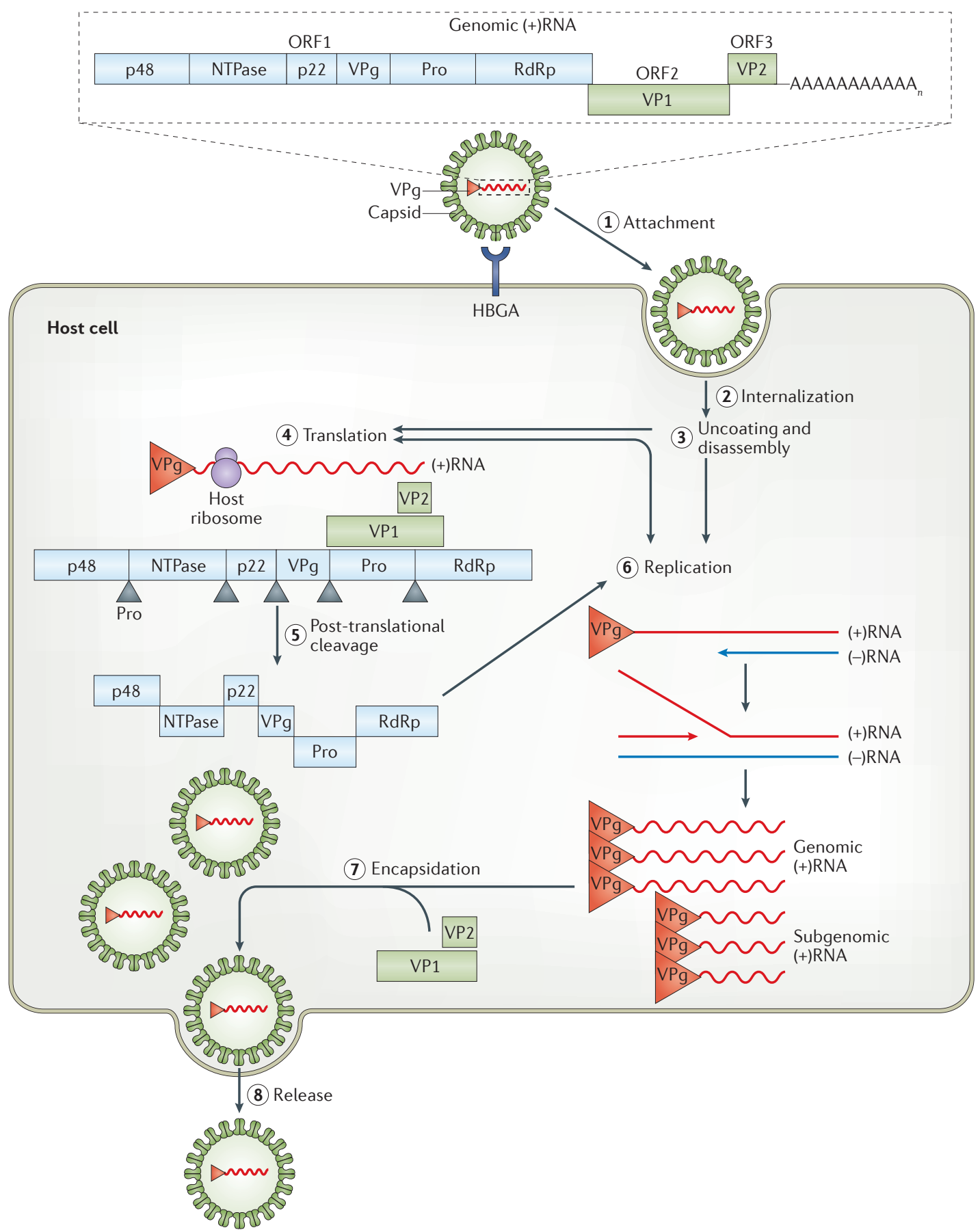

Figure 1 | The composition and life cycle of human noroviruses. The norovirus genome has three ORFs, which encode a polyprotein - encompassing six individual non-structural proteins - and the structural proteins VP1 and VP2. The genome, in the form of a positive-sense RNA strand ((+)RNA), is encapsulated in a capsid that is formed by VP1 and VP2. The capsid attaches to the cell surface through interactions between VP1 and host histo-blood group antigens (HBGAs) (step 1), and is subsequently internalized, uncoated and disassembled (steps 2,3). The (+)RNA is then transcribed and translated in the cytoplasm of the host cell. Translation is mediated by host translation factors that are recruited by the non-structural virus protein $\mathrm{VPg}$, which covalently binds to the $5^{\prime}$ end of the genome (step 4). The polyprotein that is encoded by ORF1 is post-translationally cleaved (step 5) by the virus-encoded protease, Pro (also known as NS6 or 3C-like), into individual proteins: p48 (also known as NS1/2 or N-term), NTPase (also known as NS3 or 2C-like), p22 (also known as NS4 or 3A-like), VPg, Pro and RNA-dependent RNA polymerase (RdRp). During genome replication, (+)RNA is transcribed into negative-sense RNAs ((-)RNAs), which are used as templates for the synthesis of new genomic and subgenomic (+)RNAs, respectively (step 6). Subgenomic (+)RNAs contain only ORF2 and ORF3, and are used for the production of VP1 and VP2. During encapsidation (step 7), genomic - and possibly subgenomic - (+)RNAs are packaged into new virions, which are subsequently released from the infected host cell (step 8), although the mechanism by which release occurs remains largely unknown. 


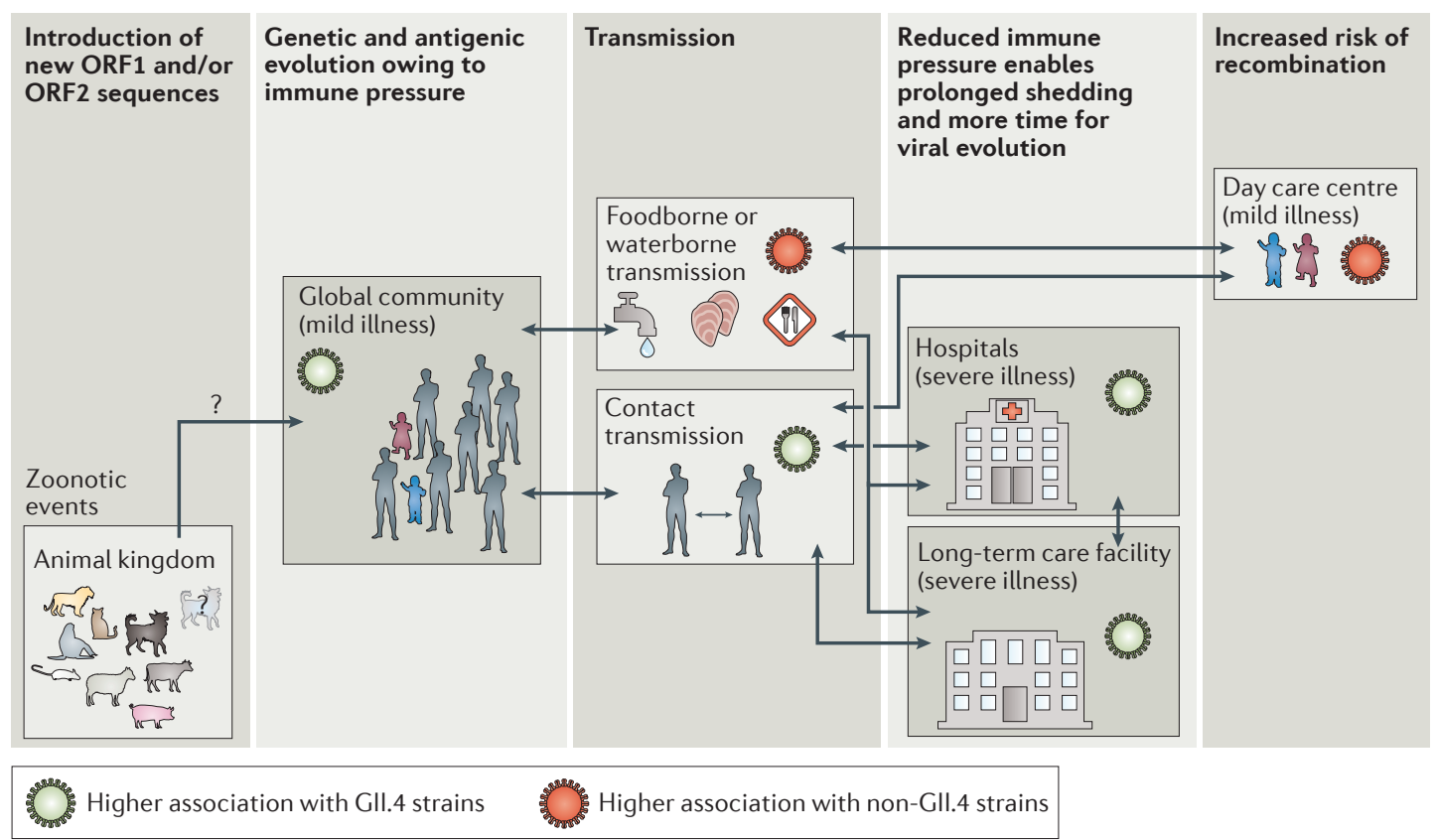

Figure 2 | Norovirus epidemiology in the community. Modes of transmission, severity of illness and evolutionary pressures all contribute to the risk that is posed by norovirus infections and can vary between strains. Although not yet reported, it is possible that zoonotic events result in the introduction of new ORF1 and/or ORF2 sequences in human noroviruses. Studies have shown a high rate of genetic and antigenic evolution for norovirus strains of the Gll.4 genotype (the genotype most associated with disease), notably antigenic variation that is driven by the selective pressure of the host immune response. These strains are also associated with contact transmission, whereas norovirus strains of non-Gll.4 genotypes are more commonly associated with foodborne or waterborne transmission. In hospitals and long-term care facilities, the circulation of Gll.4 norovirus strains in populations of immunocompromised patients can result in prolonged virus shedding, which allows more time for the viruses to undergo genetic evolution. The high diversity of non-Gll.4 genotypes that are found in day care centres increases the risk of recombination.

Lewis blood group A blood group system based on the expression of glycoproteins called Lewis antigens.

Mesoamerican

From a region that extends south and east from central Mexico to include parts of Guatemala, Belize, Honduras and Nicaragua have been detected in humans, with higher levels in veterinarians than in the general population ${ }^{39,40}$.

Although we have gained important insights into the routes of transmission and the norovirus genotypes that are associated with each route, many details about norovirus transmission remain unknown, especially details regarding the origin of newly emerging strains. For example, genogroup GI and GII strains with previously unknown ORF1 and ORF2 sequences have been detected in the human population. The fact that they have not been seen before can perhaps be explained by undersampling of the human population, including immunocompromised individuals. Alternatively, these strains may have been transmitted to humans after previously circulating in other species.

\section{Pathogenesis}

The pathogenesis of noroviruses is characterized by heterogeneous host-virus interactions, such that not all individuals are equally susceptible to norovirus infections and not all genotypes of norovirus are equally pathogenic ${ }^{41-43}$ (BOX 2). But what underlies these differences? Despite major advances in our understanding of the pathogenesis of human noroviruses, many aspects of virulence and host susceptibility remain unknown. However, animal and volunteer studies, together with patient and in vitro data, have shown that several key factors seem to have an important role in norovirus pathogenesis. These factors include viral attachment to HBGAs, the tissue and cellular tropism of a strain, the host immune responses to infection, and the bacterial microbiota of the host.

Attachment to histo-blood group antigen variants. HBGAs are glycans that are expressed on the surface of specific cells - and present in saliva and other bodily secretions - and are determinants of both the ABO blood group and Lewis blood group systems ${ }^{44}$ (FIG. 3). In certain cell types, $\alpha(1,2)$-fucosyltransferase 2 (FUT2; also known as galactoside 2- $\alpha$-L-fucosyltransferase 2 ) adds a fucose group to precursors of HBGAs, generating $\mathrm{H}$ HBGAs, and subsequent reactions generate A and B HBGAs. The binding specificity of norovirus VP1 to different HBGAs differs among norovirus genotypes and genogroups ${ }^{19}$, resulting in differences in the susceptibility of human individuals to specific strains of norovirus $^{42,43}$. Individuals who lack FUT2 are known as non-secretors, as $\mathrm{A}, \mathrm{B}$ and $\mathrm{H}$ HBGAs are not present in the bodily secretions of these individuals ${ }^{45}$. Around $20 \%$ of Northern Europeans are non-secretors ${ }^{42}$, and children of Mesoamerican ancestry are more likely to be secretors than children of European or African ances$\operatorname{try}{ }^{46}$. Non-secretors have been shown to be less susceptible to infection with several GI and GII strains of 


\section{Box 2 | Clinical symptoms of norovirus infections}

The clinical symptoms of norovirus infections include vomiting, abdominal cramps, fever, the presence of mucus in stool, watery diarrhoea, headache, chills and myalgia, and they manifest after an incubation period of 12-48 hours. However, the mechanisms by which human noroviruses infect the intestinal tract and cause disease are not fully understood. An acute onset of vomiting can occur on the first day following exposure to the virus ${ }^{2,29}$ and has been suggested to be the result of a delay in gastric emptying ${ }^{113}$. Diarrhoea is the most frequently reported symptom for norovirus infections, and virus shedding in faeces begins 18 hours or more after infection ${ }^{2}$. Biopsies have shown that, in immunocompromised patients, the pathology of norovirus infections includes villous blunting, a slight increase in the number of apoptotic epithelial cells at the luminal surface of the duodenum and a more substantial increase in the number of intraepithelial lymphocytes ${ }^{114}$. In healthy individuals, norovirus infections have been shown to result in villous blunting and infiltration of mononuclear cells at the jejunum ${ }^{115}$. Ex vivo culture of biopsies from infected humans has shown that norovirus infection leads to epithelial-barrier dysfunction that manifests as an increase in the number of both cytotoxic intraepithelial lymphocytes and apoptotic epithelial cells, and a decrease in protein abundance at tight junctions, compared with levels in controls ${ }^{116}$. This dysfunction of the epithelial barrier possibly contributes to the diarrhoea that is associated with norovirus infections, through a leak flux mechanism in which ions and water diffuse from subepithelial capillaries into the intestinal lumen ${ }^{116}$.

Not all norovirus genotypes seem to be equally pathogenic; for example, compared with other strains, Gll.4 strains are more often associated with vomiting ${ }^{41}$, a longer duration of diarrhoea and a greater number of diarrhoea stools ${ }^{117,118}$. Some studies suggest that infections with Gll.4 strains also result in a higher level of virus shedding than infections with other strains ${ }^{119}$. However, the observed heterogeneity in virus shedding might be more related to the underlying health status and age of infected individuals than the virus genotype ${ }^{32}$.

However, norovirus infection does not necessarily lead to clinical symptoms, as shedding can be detected in $7 \%$ of asymptomatic individuals in developed countries ${ }^{28}$, and in a much higher proportion in resource-limited countries ${ }^{110}$.

Villous blunting

Flattening of the intestinal villi

as a result of damage or injury.

Host cell tropism

The specificity of a virus for a

particular host cell type.

Virus-like particles

(VLPS). Particles that resemble

natural viral particles and

contain viral structural

proteins, such as the envelope

or capsid protein, but do not

contain genetic material from the virus.

Goblet cells

Specialized epithelial cells that secrete mucus and are found in the mucous membranes of the stomach, intestines and respiratory passages.

Lamina propria

The layer of connective tissue that underlies the epithelium of a mucous membrane.

Brunner's glands Tubular submucosal glands found in the duodenum. norovirus ${ }^{42,43,47}$. It should be noted that although $\mathrm{A}, \mathrm{B}$ and $\mathrm{H}$ HBGAs correspond to the $\mathrm{A}, \mathrm{B}$ and $\mathrm{O}$ blood groups, the $\mathrm{ABO}$ blood group system is independent of secretor status, as FUT1 rather than FUT2 synthesizes these HBGAs for expression on the surfaces of erythrocytes $^{48}$. However, individuals with blood type B or blood type $\mathrm{AB}$ were also found to be less susceptible to infection with certain strains of norovirus than individuals with blood type A or blood type $\mathrm{O}^{42,49}$.

Differences in the expression of HBGAs have a major effect on the susceptibility of individuals to norovirus infections and on the pathogenesis of norovirus strains, as shown in several studies, including a human challenge study with a norovirus GII.4 Farmington Hills 2002 strain. In healthy adults, challenge resulted in the infection of $70 \%$ of those individuals with a functional FUT2; of these, $57 \%$ developed symptoms of infection. By contrast, only a single individual (6\%) was infected in the group without a functional FUT2, and this individual displayed minimal disease $\mathrm{e}^{50}$. The HBGA specificities of norovirus genotypes, including GII.4 strains, have been comprehensively reviewed elsewhere ${ }^{51,52}$.

Similarly to human noroviruses, animal noroviruses use carbohydrates as attachment factors. Murine norovirus 1 (MNV-1) binds to terminal sialic acids, but the exact glycan structures that are bound by murine noroviruses differ in a strain-dependent manner ${ }^{53}$. Bovine noroviruses bind to $a$-galactose as a terminal residue of carbohydrates ${ }^{54}$, whereas canine noroviruses belonging to genogroups GIV and GVI recognize $\alpha(1,2)$-fucose-containing $\mathrm{H}$ and $\mathrm{A} \mathrm{HBGAs} \mathrm{H}^{55}$. Differences in the binding of carbohydrates between animal and human noroviruses are likely to have a role in the observed species specificity. In addition to HBGAs, it is possible that other factors may contribute to the attachment and/or entry of noroviruses into host cells. However, if such factors exist, their roles in norovirus pathogenesis have yet to be characterized.

Tropism in the human host. The host cell tropism of noroviruses has been studied using virus histochemistry, in which tissue sections are incubated with whole viruses or virus-like particles (VLPs), which are subsequently stained with specific antibodies. However, some discrepancies in the results produced by these studies have been noted. Incubation of fresh human ileum biopsies with VLPs from GII.6 or GII.1 strains resulted in the viruses binding to epithelial and goblet cells $^{56}$. Similarly, using formalin-fixed jejunal mucosae from individuals with different blood types, staining at the luminal surface of the epithelium was observed, without significant differences in staining between VLPs from GI.1, GII.2 and GII.6 strains ${ }^{57}$. By contrast, in another study, incubation with GII.4 viruses revealed attachment to cells of the lamina propria and Brunner's glands, but not to cells of the luminal epithelial surface of human duodenal tissue, which suggests that epithelial cells are not the primary targets ${ }^{58}$. It remains to be seen whether these discrepant observations reflect true differences in host cell tropism, possibly between different strains of norovirus and/or different hosts; if such differences do exist, further study will be required to establish how they might relate to differences in pathogenesis.

Until very recently, it had proved difficult to establish a cell culture system for human noroviruses ${ }^{59}$, and this had raised the question of what cell types noroviruses infect in vivo. However, mouse studies have shown that murine noroviruses replicate in macrophages, dendritic cells and B cells in vivo, and in several mouse macrophage and B cell lines in vitro ${ }^{14,22}$. Furthermore, human noroviruses infect macrophage-like cells in the livers and spleens of immunocompromised mice ${ }^{60}$. In addition to mice, chimpanzees and pigs have been experimentally infected with human noroviruses. In chimpanzees, the viruses were primarily detected in cells in the duodenal lamina propria that express DC-SIGN (also known as CD209) and in B cells ${ }^{61}$. In pigs, viral replication was detected only in duodenal and jejunal enterocytes ${ }^{37}$. Collectively, these findings indicate a role for immune cells as host cells in human norovirus infections.

Host immune response. Why norovirus infections can result in severe complications and chronic infections in certain high-risk groups is not fully understood, as the factors that offer protection to the host during infection with human noroviruses are not fully known. For murine noroviruses, components of the adaptive immune system, including B cells, $\mathrm{CD} 4^{+} \mathrm{T}$ cells and $\mathrm{CD}^{+} \mathrm{T}$ cells, are required for efficient viral clearance from the intestine and intestinal lymph nodes ${ }^{62}$. 

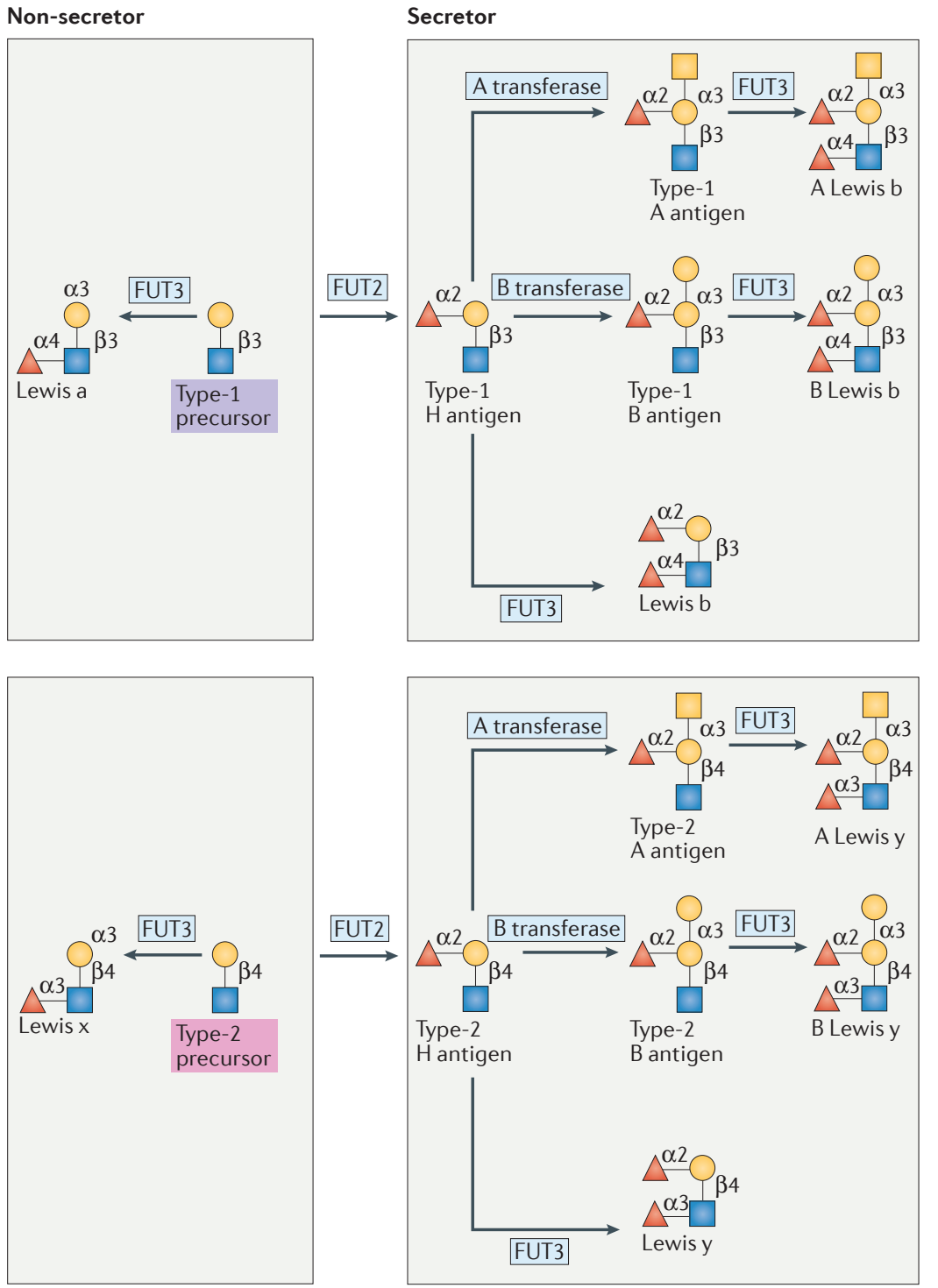

$\bigcirc$ Gal $\square$ GlcNAc $\square$ GalNAc $\triangle$ Fuc

Figure 3 | Synthesis of histo-blood group antigens. Host proteins known as histo-blood group antigens (HBGAs) provide attachment sites for noroviruses on the host cell membrane. Type- 1 and type-2 HBGA precursors are modified by $\alpha(1,2)$-fucosyltransferase 2 (FUT2) to produce H HBGAs, which are further modified by $A$ and $B$ transferases to produce A and B HBGAs. Lewis HBGAs are determinants of the Lewis blood group system and are produced when HBGAs are modified by a $(3,4)$-fucosyltransferase (FUT3; also known as galactoside 3(4)-L-fucosyltransferase). Individuals without a functional FUT2 are referred to as non-secretors, owing to the absence of A, B and H HBGAs in bodily secretions, whereas individuals with a functional FUT2 are referred to as secretors. Fuc, fucose; Gal, galactose; GalNAc, N-acetylgalactosamine; GlcNAc, N-acetyleglucosamine. Figure modified with permission from REF. 47, Springer.

\section{DC-SIGN}

(Dendritic-cell-specific

ICAM3-grabbing non-integrin). A C-type lectin receptor present on the surface of macrophages and dendritic cells.
In addition to the adaptive immune system, the innate immune system seems to have an important role in the clearance of infection. For example, pathogenesis studies of MNV-1 have revealed that interferon-mediated innate immunity is important for the clearance of infection and the reduction of pathogenicity ${ }^{63}$. The important role for interferons in norovirus infections is further highlighted by studies showing that pigs with impaired innate immune responses can be infected with a lower dose of a human norovirus than pigs with normal innate immune responses ${ }^{64,65}$.

Early studies of infection in humans suggested that the acquisition of protective immunity to noroviruses is short term ${ }^{66}$, but more recent reports indicate that protective immunity is longer lasting than initially thought ${ }^{67,68}$. Owing to the historical lack of a cell culture system for the study of norovirus replication, virus neutralization has not been measured directly and the measurement of the inhibition of VLP binding to HBGAs has instead been used as a surrogate assay ${ }^{69}$. In challenge studies in humans and chimpanzees, increased serum titres of antibodies that inhibited VLP binding to HBGAs correlated with a reduction in the rate of infection and in disease severity ${ }^{61,70}$. In human challenge studies, an early mucosal immunoglobu$\operatorname{lin} \mathrm{A}(\operatorname{Ig} \mathrm{A})$ response was associated with protection against norovirus infection ${ }^{43}$. Furthermore, pre-existing norovirus-specific IgA in saliva and norovirus-specific memory IgG cells were associated with protection from gastroenteritis ${ }^{71}$; moreover, pre-existing faecal norovirus-specific IgA was associated with a reduction in peak viral load, and the magnitude of faecal levels of IgA measured 1 week after infection correlated with a shorter duration of shedding ${ }^{71}$. In conclusion, these findings support a role for host immune responses in reducing the viral load, the duration of virus shedding and the severity of disease.

The role of bacteria. Recent studies have identified three mechanisms by which bacteria in the host microbiota might influence human norovirus infections: by providing additional HBGAs for the norovirus to bind to, by influencing the expression of host HBGAs in the gut and by modulating host immunity. In an in vitro study, B cells were incubated with faecal samples from patients who were infected with a GII.4 strain ${ }^{14}$. Filtration of the patient samples resulted in a reduction of viral replication compared with levels in non-filtered samples, indicating that the bacteria in the sample may have a role in facilitating norovirus infection of the B cells. The authors of the study suggested virus attachment to bacterial HBGA-like sugars as a possible mechanism for an interaction between noroviruses and bacterial cells. Several species of bacteria that are found in the host microbiota are able to express HBGA-like sugars, including Enterobacter cloacae, Escherichia coli and Helicobacter pylori ${ }^{14,72,73}$. E. cloacae expresses $\mathrm{H}$ HBGA-like sugars, which the authors showed could be bound by GII. 4 viruses ${ }^{14}$. Furthermore, incubation of the norovirus with inactivated E. cloacae or synthetic H HBGA restored the infectivity of filtered patient samples ${ }^{14}$. The molecular mechanism by which bacterial HBGA-like sugars facilitated norovirus infection of B cells in the in vitro assay is not known, but one possibility is that the binding of viral particles to HBGA-like sugars that are expressed on the surface of bacterial cells - instead of, or in addition to, HBGAs on the host cells - enables uptake of the virus into the host cells ${ }^{14}$. 
In addition to the expression of their own HBGAlike sugars, $H$. pylori strains might influence norovirus infection through the modulation of host HBGA expression: the presence of $H$. pylori strains that express the virulence factor CagA is associated with the expression of HBGAs in non-secretor individuals, thereby potentially expanding the host range for noroviruses ${ }^{74}$. Finally, bacteria in the host microbiota may contribute to norovirus pathogenesis by the modulation of host immunity. Depleting the microbiota of mice using antibiotics resulted in lower viral titres ${ }^{14}$, revealing a role for the host microbiota in murine norovirus infections. In a second study, antibiotics that deplete the bacterial microbiota were also effective in the prevention of persistent norovirus infection $^{75}$. Surprisingly, depletion of the microbiota did not affect systemic viral replication, but acted specifically in the intestine. The interferon- $\lambda$ receptor, as well as the transcription factors signal transducer and activator of transcription 1 (STAT1; which is regulated by interferon signalling) and interferon regulatory factor 3 (IRF3; which regulates interferon-encoding genes), was essential for the prevention of viral persistence in antibiotic-treated mice, leading the authors to suggest that the bacterial microbiota hampers the efficacy of interferon- $\lambda$ signalling, which is required for the clearance of persistent murine norovirus infection by the innate immune system ${ }^{75}$.

\section{Genetic diversity and evolution}

Viruses in the genus Norovirus can be found in a wide range of hosts, such as humans, rodents, felines, canines, sea lions, pigs, sheep, cattle and bats ${ }^{15,76,77}$ (FIG. 4). The nucleotide sequences of the genomes of different norovirus genogroups share only $51-56 \%$ similarity with one another, and the diversity between genogroups is even higher when comparing only ORF2 sequences rather than whole genomes ${ }^{19,78}$. Despite frequent recombination and possible differences in selection pressures between ORF1 and ORF2, the phylogeny of ORF1 has a similar topology and a similarly high genetic diversity to the phylogeny of ORF2 (REF. 15) (FIG. 4). Intriguingly, some outbreaks are caused by strains of norovirus that are genetically similar or identical to strains that were isolated 10-15 years earlier, which raises questions about the reservoirs in which these viruses are maintained between outbreaks ${ }^{7}$. Overall, the evolution of noroviruses is complex, and a better understanding of the genetic and antigenic evolution of the GII.4 genotype, and indeed all norovirus genotypes, is of crucial importance for the development of vaccines and treatment strategies.

Evolution of GII.4 strains. Surveillance studies have shown that globally circulating GII.4 strains are frequently replaced by newly emerged antigenically divergent GII.4 strains, which indicates that an immunogenic pressure influences the evolution of noroviruses, at least for the GII.4 genotype ${ }^{19}$ (FIG. 5a). Importantly, the emergence of antigenically divergent GII.4 strains coincides with an increase in norovirus outbreak activity ${ }^{79}$. Bioinformatic analyses and in vitro assays have shown that GII.4 strains have high rates of mutation and evolution, which probably facilitate the emergence of these antigenically divergent strains ${ }^{80}$. Molecular epidemiology of GII.4 isolates collected across the globe showed that some GII.4 lineages that are able to cause widespread regional epidemics were nevertheless geographically limited ${ }^{8}$. The failure of these epidemics to spread throughout the world could be due to differences in the genetic and microbial make-up of the host or differences in the previous exposure of host populations to noroviruses. Recently, major outbreaks of the newly emerged GII.P17-GII.17 strain of norovirus have occurred in some parts of Asia $^{17}$, where it has replaced the previously circulating GII.4 Sydney 2012 strain. Although the GII.P17-GII.17 genotype has also been detected in gastroenteritis cases in Europe, the United States and Australia, it has not yet replaced GII.4 strains in these regions ${ }^{17}$.

Since 1995, six antigenically variant GII.4 strains have resulted in pandemics: US 1995/96, Farmington Hills 2002, Hunter 2004, Den Haag 2006b, New Orleans 2009 and Sydney 2012 (REF. 81). The emergence of the Farmington Hills antigenic variant in 2002 coincided with an increase in the number of reported norovirus outbreaks ${ }^{82}$, which was confirmed by phylodynamic reconstruction to reflect a true increase in infections rather than reporting bias ${ }^{79}$.

The amino acid positions in VP1 that are associated with antigenic change are located in the $\mathrm{P}$ domain ${ }^{83}$. As the P domain contains the HBGA-binding site and as only a few amino acid mutations are required to change the binding specificity of this site, antigenic change may correlate with a change in HBGA-binding specificity; indeed, the HBGA-binding specificities of GII.4 noroviruses have changed over time (FIG. 5a), which may contribute to the high prevalence of the GII.4 genotype ${ }^{19,84}$. The variation in HBGA-binding preferences between GII.4 strains may have implications for the susceptibility of the local host population towards each variant, according to the prevalence of each HBGA in the given population.

Evolution of non-GII.4 strains. A comparison of the evolution of strains from non-GII.4 genotypes and GII.4 strains suggests that non-GII.4 strains are subject to less adaptive pressure. Although less prevalent than GII.4 strains, GII.3 strains are frequently detected in patient samples, particularly in children, and evolve at a rate of $4.16 \times 10^{-3}$ nucleotide substitutions per site per year, which is similar to the rate of evolution of the GII.4 and GI strains ${ }^{85,86}$. However, despite similar nucleotide substitution rates, the accumulation of amino acid mutations is much lower for GII.3 strains than for GII.4 strains ${ }^{85}$, which is indicative of a more limited immunogenic pressure on the GII.3 strains.

GII.2 strains also exhibit only limited antigenic evolution, although some evidence for genetic drift has been observed $^{87,88}$. In an assay using antibodies elicited in human volunteers that were infected with Snow mountain virus (strain GII.2 1967), HBGA binding to VLPs 

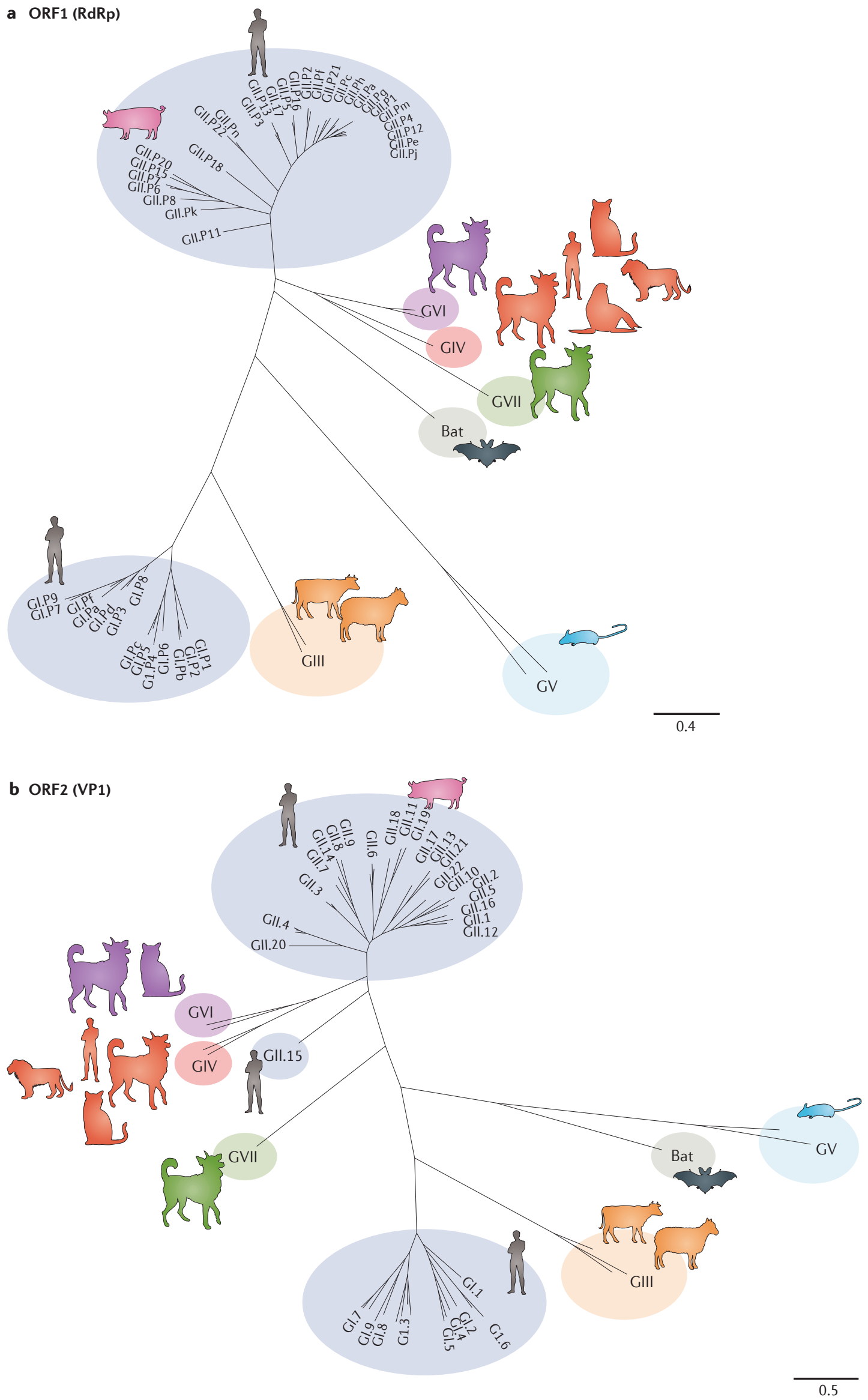
4 Figure 4 | ORF1 and ORF2 phylogenies. Two regions of the norovirus genome are used to classify strains of norovirus: the region of ORF1 that encodes the RNA-dependent RNA polymerase (RdRp), and ORF2, which encodes the structural capsid protein VP1. Genetic diversity and frequent recombination events between ORF1 and ORF2 have resulted in phylogenetic topologies that, although similar, are not identical, as shown in unrooted maximum likelihood trees estimated for ORF1 (part a) and ORF2 (part b) sequences of all norovirus ORF1 and ORF2 genotypes ${ }^{15}$. Owing to the frequent occurrence of recombination events between ORF1 and ORF2 sequences, a dual nomenclature for norovirus classification using both sequences encoding RdRp and sequences encoding VP1 has been proposed ${ }^{120}$. Note that the nomenclature of genogroups GIV, GVI and GVII has not been consistent: genogroups GIV and GVI were initially classified as a single genogroup, which was known as genogroup GIV, and norovirus strains in genogroup GVII have also been classified in the past as belonging to genogroup GVI. However, we have chosen to use seven genogroups, as proposed by Vinjé é $^{15}$; this reclassification is based on amino acid divergence. The scale bar reflects the number of nucleotide substitutions per site. Part b is modified from J. Clin. Microbiol., 2015, 53, 373-381, http://dx.doi.org/10.1128//CM.01535-14 and amended with permission from American Society for Microbiology.

derived from the infecting strain was more effectively inhibited than VLPs derived from GII.2 strains that have circulated more recently, although HBGA binding to these VLPs was nevertheless inhibited to some degree $^{88}$.

Evolution by recombination. Recombination of human noroviruses is frequently observed and is thought to be an important mechanism by which genetic diversity is generated (FIG. 5b,c). Recombination between human noroviruses most commonly occurs near the junction of ORF1 and ORF2 and can be associated with antigenic shift $^{89}$. A less common site of recombination is located at the junction of ORF2 and ORF3 (REF. 90).

GII.3 strains cluster into four lineages, each of which is associated with a different ORF1 genotype. The emergence of each lineage was accompanied by an increase in relative genetic diversity, suggesting that recombination results in a higher rate of evolution, which may offer a temporary selective advantage $^{91}$. This finding illustrates the probable role of recombination events in altering the efficiency of virus replication. For human noroviruses, the challenge of developing a cell culture system had historically prevented the investigation of replication efficiencies. However, in recent years, replicon systems have been developed in human cell lines; together with studies of murine noroviruses and biochemical studies, cellbased systems have provided new insights into the mechanisms of norovirus replication ${ }^{18,92-94}$. For example, interactions between VPg (also known as NS5) and RNA-dependent RNA polymerase (RdRp; also known as NS7), both encoded by ORF1, were found to be crucial for the replication of norovirus ${ }^{94}$; however, these interactions were also shown to be specific for noroviruses that infect the same species, as was the enhancement of RdRp activity by VP1 (REF. 95). The interactions between the various proteins that are required for replication probably limit successful recombination between viruses that infect different species and possibly between human noroviruses from different genogroups or genotypes.

\section{Treatments and interventions}

Clinical intervention efforts for norovirus infection are hampered by the lack of a licensed vaccine, despite important advances in vaccine development, and limited evidence for the success of the antiviral treatment options that are currently available. Several individuals who were chronic shedders have been successfully treated with oral human immunoglobulin, although in some patients treatment did not result in clearance of the virus ${ }^{96,97}$. Additional studies will be required to determine whether the route of administration and/or the levels of antibodies that are specific to the infecting strain of norovirus are important factors in the success rate of human immunoglobulin treatment. The ability of immunoglobulin to limit infection was also seen in a mouse model of norovirus infection following intraperitoneal administration of immunoglobulin ${ }^{98}$. Another strategy that may clear norovirus infections in immunocompromised patients is the partial restoration of the immune system, whether by reducing, temporarily discontinuing or changing immunosuppressive drugs $^{99}$; however, this should be done with caution, and is not possible for all patients.

Antivirals, including nitazoxanide, ribavirin and interferons, have been shown to inhibit norovirus replication in cell culture-based replicon systems, mouse models or infected human individuals ${ }^{65,100-104}$. Oral treatment with nitazoxanide, an agent that has broad antimicrobial activity, resulted in clinical resolution of acute gastroenteritis in a patient who was chronically infected, although asymptomatic shedding was observed for another month ${ }^{101}$. Nitazoxanide also reduced the duration of symptoms in a small randomized, doubleblind, placebo-controlled clinical trial ${ }^{100}$. Two chronically infected immunocompromised individuals were successfully treated with oral ribavirin, which is a broad-spectrum antiviral agent, although a similar treatment was unsuccessful in two other patients ${ }^{102}$. In pigs, oral treatment with natural human interferon- $\alpha$ reduced or curtailed virus shedding during the treatment period compared with levels and duration of shedding in untreated pigs, but virus shedding increased to normal levels after the termination of treatment ${ }^{65}$. In mice, oral treatment with interferon- $\lambda$ cured persistent norovirus infections ${ }^{103}$. However, no clinical data are currently available relating to the effect of interferon treatment on the replication of norovirus in humans.

Historically, the development of a norovirus vaccine has been hampered by the lack of a small-animal model and a cell culture system, both of which have been described only recently, and licensed vaccines are not yet available $e^{21}$. Nevertheless, the first norovirus vaccines have now completed Phase I and Phase II clinical trial $^{21}$; these vaccines are based on VLPs of the GI.1 genotype or, in the case of the bivalent vaccine, contain both GI.1-derived VLPs and VLPs based on the consensus sequence of several GII.4 variants ${ }^{21}$. The clinical trials showed an induction of antibody responses that occurred regardless of whether the vaccine was administered intramuscularly, orally or intranasally ${ }^{105}$. In a clinical trial with healthy volunteers, intramuscular 


\section{REVIEWS}

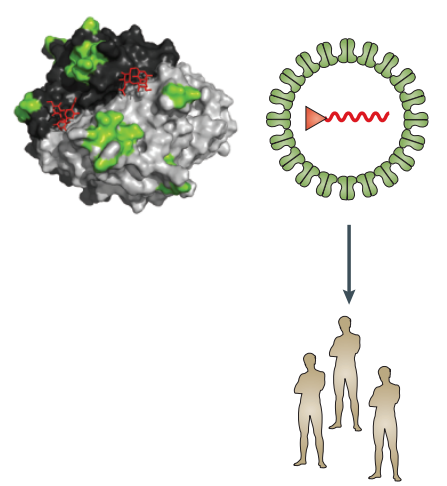

Infection of susceptible population

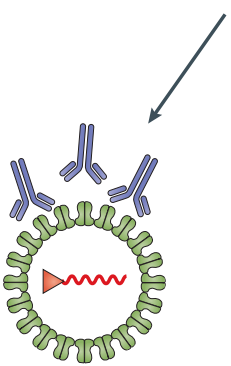

Host population develops antibodies
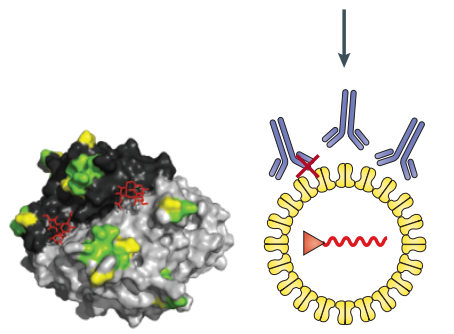

Variation in virus genome creates antigenic variants

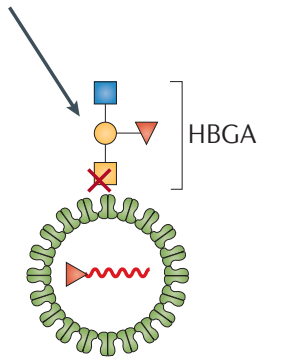

Host population varies in receptor expression

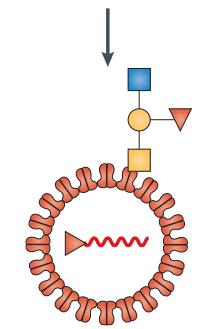

Variation in virus genome creates differential binders b

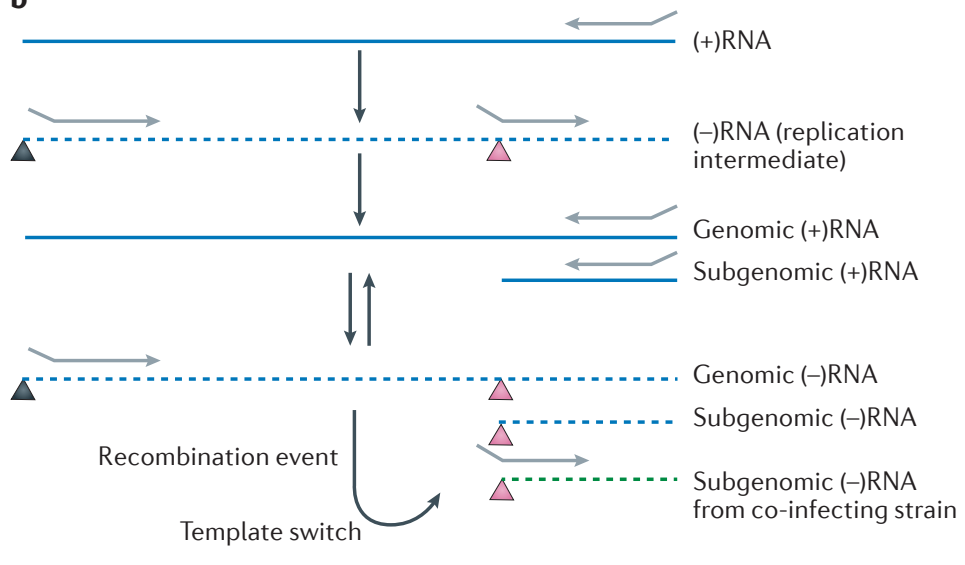

Recombinant (+)RNA

c

Co-infecting virus strain 1

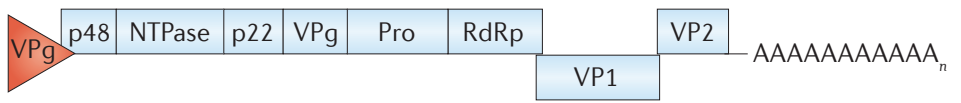

Co-infecting virus strain 2

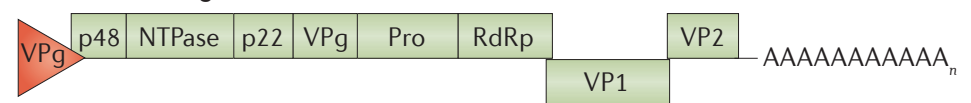

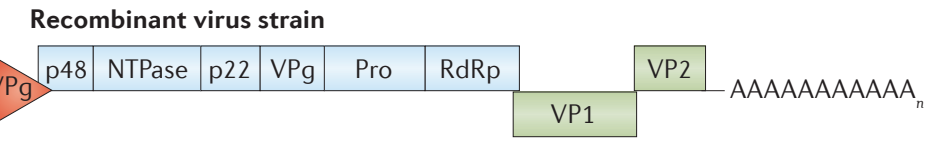

Figure 5 | Mechanisms of norovirus evolution. a | Antigenic variation occurs in the $\mathrm{P}$ domain of norovirus capsid protein VP1. A dimer of the P domain from a Gll.4 Sydney 2012 norovirus, with antigenic sites A, D and E (green), is shown bound to histo-blood group antigens (HBGAs; red), which are host cell attachment factors for noroviruses; individual monomers of the P domain are depicted in black and grey (RCSB Protein Data Bank (PDB) entry 4WZT). The emergence of novel antigenic variants of noroviruses is driven by antigenic drift, which enables immune evasion (left pathway), or by heterogeneity in the expression of HBGAs in the human population (right pathway). For example, a comparison of the Gll.4 Orleans 2009 strain and the more recent Gll.4 Sydney 2012 strain reveals amino acid substitutions in antigenic sites (yellow) $)^{83} \cdot \mathbf{b} \mid$ Norovirus recombination can occur by a simple mechanism. Genomic positive-sense viral RNA ((+)RNA) is transcribed into negative-sense intermediates ((-)RNA) that provide templates for the transcription of genomic and subgenomic (+)RNA ${ }^{89}$. Recombination occurs when the RNA-dependent RNA polymerase (RdRp) initiates (+)RNA synthesis at the promoter (black triangle) located at the $3^{\prime}$ end of a (-)RNA but stalls at the subgenomic promoter (pink triangle) and then switches template to the subgenomic (-)RNA of a co-infecting virus. c | Following recombination, a recombinant virus strain is produced with a viral RNA that has a novel combination of ORF1 and ORF2-ORF3. Part b is modified with permission from REF. 89, CDC.

Heterologous

cross-protection

Protection conferred on a host by inoculation with one strain of a microorganism (or a component of the strain) that prevents infection when the host is later challenged with a different strain. vaccination with bivalent VLPs did not significantly reduce the incidence of protocol-defined illness after challenge with a GII.4 strain of norovirus; however, the vaccination was able to reduce the incidence and severity of vomiting and diarrhoea ${ }^{106}$. As an alternative strategy to vaccination with VLPs, intranasal vaccination with a vaccine derived from only the VP1 P domain, rather than the entire viral capsid, resulted in partial heterologous cross-protection in gnotobiotic pigs that were infected with noroviruses of the GII.4 genotype ${ }^{107}$.
The development of vaccines is complicated by the high degree of genetic diversity and the high rate of antigenic evolution of noroviruses. These complications may mean that it will be necessary to update the vaccine every 2-3 years, as is the case for influenza vaccines, unless a vaccine is able to induce a broadly reactive antibody response that remains effective against new antigenic variants. Encouragingly, the bivalent vaccine containing GI.1 VLPs and GII.4 VLPs produced broadly reactive antibody responses in humans in a 
clinical trial, providing heterologous cross-protection to vaccine and non-vaccine variants ${ }^{69}$. In mice, a trivalent vaccine that combined norovirus GII.4 and GI.3 genotypes with the rotavirus VP6 genotype also induced a broadly reactive immune response that provided heterologous cross-protection ${ }^{21,108}$. Although the development of norovirus vaccines is currently focused on the most prevalent genotypes, the recent emergence of GII. P17-GII.17 noroviruses in Asia should act as a warning that future risks from norovirus outbreaks might arise from genotypes other than those currently targeted for vaccine development. As much remains to be understood about the factors that drive norovirus epidemiology, assumptions about which genotypes are likely to be responsible for future outbreaks may not be reliable. Therefore, the future efficacy of vaccines against noroviruses may rely on the development of vaccines that are able to elicit a broadly reactive immune response or on an improved understanding of norovirus evolution.

\section{Outlook}

In this Review, we have described how the natural barriers that limit the potential for norovirus transmission within the human population are being reduced by factors such as human population growth, international travel and increasing globalization of food distribution. Furthermore, we have discussed the high rate of antigenic evolution observed in noroviruses, notably in genotype GII.4, and how antigenic changes may alter viral binding specificities, which may result in more efficient transmission. Finally, we have outlined the advances that have been made in the clinical treatment and prevention of norovirus infections, efforts which currently suffer from a dearth of proven antiviral treatment options and a lack of a licensed vaccine. In addition to these challenges, the threat posed by noroviruses is further enhanced by an increase in the number of immunocompromised patients that has arisen as the general population ages and the number of patients in receipt of solid-organ and haematopoietic stem cell transplants - and the survival time of these patients - increases. The increased burden thus posed by noroviruses on a global scale warrants an international effort to understand the ecology, molecular epidemiology, evolution, pathogenesis and clinical impact of noroviruses.

Key priorities in addressing the challenges posed by noroviruses are the development of an antiviral that can effectively prevent or treat norovirus infections in high-risk individuals, for whom the severity of disease is greatest, and the development of a licensed vaccine against noroviruses. The high degree of genetic and antigenic diversity in noroviruses means that vaccines and antiviral drugs should be designed to be effective against a broad spectrum of norovirus strains. To assess the efficacy of vaccines and antivirals, it is crucial that appropriate animal model and cell culture systems are available. In this regard, the development of cell culture-based replicon systems for studying human noroviruses has enabled the study of antivirals that target viral replication, whereas assays that measure the inhibition of virus binding to HBGAs can be used to investigate neutralizing antibodies. Caliciviruses other than noroviruses have also been used as model systems to study the replication and stability of human noroviruses. However, owing to differences in host range, these viruses may not closely model the basic properties of human noroviruses, such as binding specificity, stability and determinants of replication, and are thus not ideal substitutes. Recent work has seen the establishment of both an in vitro cell culture system for studying the replication of human noroviruses in $\mathrm{B}$ cells and a mouse model for studying the replication of human noroviruses in immunocompromised mice; these tools will hopefully advance our understanding of many aspects of human noroviruses, ranging from the molecular characterization of the life cycle to the development of improved vaccines ${ }^{14,60}$.

In addition to immunocompromised mice, ferrets might also be expected to be useful as a laboratory animal model of norovirus infection, as prolonged shedding of an influenza virus in immunocompromised patients was mimicked in immunocompromised ferrets ${ }^{109}$. However, despite the suitability of ferrets as an animal model for many human viruses, immunocompromised ferrets do not allow the replication of GI and GII norovirus strains (M.d.G., J.v.B., M.P.G.K., K. J. Stittelaar and G. van Amerongen, unpublished observations). Human volunteer studies and studies in larger animal models are cumbersome and costly, but may provide essential information about the determinants of host range and the drivers of evolution in noroviruses.

Currently, national and international norovirus surveillance networks monitor which strains are circulating in humans throughout the year (CaliciNet in the USA; and NoroNet, as well as national networks, in Europe, South Africa, Asia, Australia and New Zealand). Surveillance of the general population, hospitals, day care centres and long-term care facilities through these networks will provide information concerning the epidemiology, pathogenicity and evolution of the different norovirus genotypes. Understanding these features of noroviruses will be crucial for the development of a long-term strategy to reduce the burden of associated disease and to prevent norovirus outbreaks in a rapidly changing world.
1. Atmar, R. L. \& Estes, M. K. The epidemiologic and clinical importance of norovirus infection. Gastroenterol. Clin. North Am. 35, 275-290 (2006).

2. Atmar, R. L. et al. Norwalk virus shedding after experimental human infection. Emerg. Infect. Dis. 14, 1553-1557 (2008).

3. Teunis, P. F. et al. Norwalk virus: how infectious is it? J. Med. Virol. 80, 1468-1476 (2008).

4. Marks, P. J. et al. Evidence for airborne transmission of Norwalk-like virus (NLV) in a hotel restaurant. Epidemiol. Infect. 124, 481-487 (2000).
5. Verhoef, L. et al. Norovirus genotype profiles associated with foodborne transmission, 1999-2012. Emerg. Infect. Dis. 21, 592-599 (2015).

6. Wikswo, M. E. \& Hall, A. J. Outbreaks of acute gastroenteritis transmitted by person-to-person contact-United States, 2009-2010. MMWR Surveill. Summ. 61, 1-12 (2012).

7. Lysen, M. et al. Genetic diversity among food-borne and waterborne norovirus strains causing outbreaks in Sweden. J. Clin. Microbiol. 47, 2411-2418 (2009).
8. Siebenga, J. J. et al. Norovirus illness is a global problem emergence and spread of norovirus Gll. 4 variants, 2001-2007. J. Infect. Dis. 200, 802-812 (2009).

9. Kroneman, A. et al. Data quality of 5 years of central norovirus outbreak reporting in the European Network for food-borne viruses. J. Public Health (Oxf) 30 82-90 (2008)

10. Harris, J. P., Edmunds, W. J., Pebody, R., Brown, D. W. $\&$ Lopman, B. A. Deaths from norovirus among the elderly, England and Wales. Emerg. Infect. Dis. 14 1546-1552 (2008). 
11. Trivedi, T. K. et al. Clinical characteristics of norovirusassociated deaths: a systematic literature review. Am. J. Infect. Control 41, 654-657 (2013).

12. Siebenga, J. J. et al. High prevalence of prolonged norovirus shedding and illness among hospitalized patients: a model for in vivo molecular evolution. J. Infect. Dis. 198, 994-1001 (2008). A study showing that noroviruses more commonly cause prolonged periods of gastrointestina illness and virus shedding than previously recognized.

13. Murata, T. et al. Prolonged norovirus shedding in infants $\leq 6$ months of age with gastroenteritis. Pediatr. Infect. Dis. J. 26, 46-49 (2007).

14. Jones, M. K. et al. Enteric bacteria promote human and mouse norovirus infection of B cells. Science 346 755-759 (2014). An important paper that describes a cell culture system for the study of human norovirus infection in $B$ cells.

15. Vinje, J. Advances in laboratory methods for detection and typing of norovirus. J. Clin. Microbiol. 53, 373-381 (2015).

16. Kroneman, A. et al. Analysis of integrated virological and epidemiological reports of norovirus outbreaks collected within the Foodborne Viruses in Europe network from 1 July 2001 to 30 June 2006. J. Clin. Microbiol. 46, 2959-2965 (2008)

17. de Graaf, M. et al. Emergence of a novel Gll.17 norovirus - end of the Gll.4 era? Euro Surveill. 20, 21178 (2015)

18. Thorne, L. G. \& Goodfellow, I. G. Norovirus gene expression and replication. J. Gen. Virol. 95, 278-291 (2014).

19. Donaldson, E. F., Lindesmith, L. C., Lobue, A. D. \& Baric, R. S. Viral shape-shifting: norovirus evasion of the human immune system. Nat. Rev. Microbiol. 8 , 231-241 (2010)

20. Vongpunsawad, S., Venkataram Prasad, B. V. \& Estes, M. K. Norwalk virus minor capsid protein VP2 associates within the VP1 shell domain. J. Virol. 87 4818-4825 (2013).

21. Debbink, K., Lindesmith, L. C. \& Baric, R. S. The state of norovirus vaccines. Clin. Infect. Dis. 58, 1746-1752 (2014).

22. Wobus, C. E. et al. Replication of norovirus in cell culture reveals a tropism for dendritic cells and macrophages. PLoS Biol. 2, e432 (2004).

23. Hoa Tran, T. N., Trainor, E., Nakagomi, T., Cunliffe, N. A \& Nakagomi, O. Molecular epidemiology of noroviruses associated with acute sporadic gastroenteritis in children: global distribution of genogroups, genotypes and Gll.4 variants. J. Clin. Virol. 56, 185-193 (2013).

24. Rodriguez-Lazaro, D. et al. Virus hazards from food, water and other contaminated environments. FEMS Microbiol. Rev. 36, 786-814 (2012)

25. Le Guyader, F. S., Atmar, R. L. \& Le Pendu, J. Transmission of viruses through shellfish: when specific ligands come into play. Curr. Opin. Virol. 2, 103-110 (2012).

26. Verhoef, L. et al. An integrated approach to identifying international foodborne norovirus outbreaks. Emerg. Infect. Dis. 17, 412-418 (2011).

27. Foodborne Disease Burden Epidemiology Reference Group 2007-2015. WHO estimates of the global burden of foodborne diseases (WHO, 2011).

28. Ahmed, S. M. et al. Global prevalence of norovirus in cases of gastroenteritis: a systematic review and meta-analysis. Lancet Infect. Dis. 14, 725-730 (2014).

29. Rockx, B. et al. Natural history of human calicivirus infection: a prospective cohort study. Clin. Infect. Dis. 35, 246-253 (2002)

30. Sukhrie, F. H. et al. Nosocomial transmission of norovirus is mainly caused by symptomatic cases. Clin. Infect. Dis. 54, 931-937 (2012).

31. Sukhrie, F. H., Siebenga, J. J., Beersma, M. F. \& Koopmans, M. Chronic shedders as reservoir for nosocomial transmission of norovirus. J. Clin. Microbiol. 48, 4303-4305 (2010).

32. Milbrath, M. O., Spicknall, I. H., Zelner, J. L., Moe, C. L. \& Eisenberg, J. N. Heterogeneity in norovirus shedding duration affects community risk. Epidemiol. Infect. 141, 1572-1584 (2013).

33. Debbink, K. et al. Within-host evolution results in antigenically distinct GII.4 noroviruses. J. Virol. $\mathbf{8 8}$ 7244-7255 (2014).

34. Mattison, K. et al. Human noroviruses in swine and cattle. Emerg. Infect. Dis. 13, 1184-1188 (2007).

35. Summa, M., von Bonsdorff, C. H. \& Maunula, L. Pet dogs - a transmission route for human noroviruses? J. Clin. Virol. 53, 244-247 (2012).
36. Souza, M., Azevedo, M. S., Jung, K., Cheetham, S. $\&$ Saif, L. J. Pathogenesis and immune responses in gnotobiotic calves after infection with the genogroup II.4-HS66 strain of human norovirus. J. Virol. 82, 1777-1786 (2008).

37. Cheetham, S. et al. Pathogenesis of a genogroup II human norovirus in gnotobiotic pigs. J. Virol. $\mathbf{8 0}$, 10372-10381 (2006)

38. Caddy, S. L. et al. Evidence for human norovirus infection of dogs in the United Kingdom. J. Clin. Microbiol. 53, 1873-1883 (2015).

39. Widdowson, M. A. et al. Detection of serum antibodies to bovine norovirus in veterinarians and the general population in the Netherlands. J. Med. Virol. 76, 119-128 (2005)

40. Mesquita, J. R. et al. Presence of antibodies against genogroup VI norovirus in humans. Virol. J. 10, 176 (2013).

41. Friesema, I. H. et al. Differences in clinical presentation between norovirus genotypes in nursing homes. J. Clin. Virol. 46, 341-344 (2009).

42. Thorven, M. et al. A homozygous nonsense mutation $(428 \mathrm{G} \rightarrow \mathrm{A})$ in the human secretor (FUT2) gene provides resistance to symptomatic norovirus (GGII) infections. J. Virol. 79, 15351-15355 (2005).

43. Lindesmith, L. et al. Human susceptibility and resistance to Norwalk virus infection. Nat. Med. 9 548-553 (2003).

44. Stanley, P. \& Cummings, R. D. in Essentials of Glycobiology 2nd edn Ch. 13 (eds Varki, A. et al.) (Cold Spring Harbor Laboratory Press, 2009).

45. Le Pendu, J., Ruvoen-Clouet, N., Kindberg, E. $\S$ Svensson, L. Mendelian resistance to human norovirus infections. Semin. Immunol. 18, 375-386 (2006).

46. Currier, R. L. et al. Innate susceptibility to norovirus infections influenced by FUT2 genotype in a United States pediatric population. Clin. Infect. Dis. 60 1631-1638 (2015)

47. Atmar, R. L. Noroviruses - state of the art. Food Environ. Virol. 2, 117-126 (2010)

48. Shirato, H. et al. Noroviruses distinguish between type 1 and type 2 histo-blood group antigens for binding. J. Virol. 82, 10756-10767 (2008).

49. Hutson, A. M., Atmar, R. L., Graham, D. Y \& Estes, M. K. Norwalk virus infection and disease is associated with ABO histo-blood group type. J. Infect. Dis. 185, 1335-1337 (2002).

50. Frenck, R. et al. Predicting susceptibility to norovirus Gll.4 by use of a challenge model involving humans. J. Infect. Dis. 206, 1386-1393 (2012).

51. Donaldson, E. F., Lindesmith, L. C., Lobue, A. D. $\&$ Baric, R. S. Norovirus pathogenesis: mechanisms of persistence and immune evasion in human populations. Immunol. Rev. 225, 190-211 (2008)

52. Tan, M. \& Jiang, X. Norovirus gastroenteritis carbohydrate receptors, and animal models. PLOS Pathog. 6, e1000983 (2010).

53. Taube, S. et al. Murine noroviruses bind glycolipid and glycoprotein attachment receptors in a straindependent manner. J. Virol. 86, 5584-5593 (2012)

54. Zakhour, M. et al. The $\alpha$ Gal epitope of the histoblood group antigen family is a ligand for bovine norovirus Newbury2 expected to prevent crossspecies transmission. PLoS Pathog. 5, e 1000504 (2009)

55. Caddy, S., Breiman, A., le Pendu, J. \& Goodfellow, I. Genogroup IV and VI canine noroviruses interact with histo-blood group antigens. J. Virol. 88, 10377-1039 (2014)

56. Murakami, K. et al. Norovirus binding to intestinal epithelial cells is independent of histo-blood group antigens. PLOS ONE 8, e66534 (2013).

57. Yazawa, S. et al. Blood group substances as potential therapeutic agents for the prevention and treatment of infection with noroviruses proving novel binding patterns in human tissues. PLOS ONE 9, e89071 (2014).

58. Chan, M. C., Ho, W. S. \& Sung, J. J. In vitro whole-virus binding of a norovirus genogroup II genotype 4 strain to cells of the lamina propria and Brunner's glands in the human duodenum. J. Virol. 85, 8427-8430 (2011).

59. Duizer, E. et al. Laboratory efforts to cultivate noroviruses. J. Gen. Virol. 85, 79-87 (2004).

60. Taube, S. et al. A mouse model for human norovirus. mBio 4, e00450-13 (2013).

A paper that describes the first mouse model for human norovirus infection.
61. Bok, K et al. Chimpanzees as an animal model for human norovirus infection and vaccine development. Proc. Natl Acad. Sci. USA 108, 325-330 (2011).

62. Chachu, K. A., LoBue, A. D., Strong, D. W. Baric, R. S. \& Virgin, H. W. Immune mechanisms responsible for vaccination against and clearance of mucosal and lymphatic norovirus infection. PLoS Pathog. 4, e1000236 (2008)

63. Karst, S. M., Wobus, C. E., Lay, M., Davidson, J. \& Virgin, H. W. STAT1-dependent innate immunity to a Norwalk-like virus. Science 299, 1575-1578 (2003).

64. Bui, T. et al. Median infectious dose of human norovirus GII.4 in gnotobiotic pigs is decreased by simvastatin treatment and increased by age. J. Gen. Virol. 94, 2005-2016 (2013).

65. Jung, K. et al. The effects of simvastatin or interferon- $\alpha$ on infectivity of human norovirus using a gnotobiotic pig model for the study of antivirals. PLOS ONE 7, e41619 (2012).

66. Parrino, T. A., Schreiber, D. S., Trier, J. S Kapikian, A. Z. \& Blacklow, N. R. Clinical immunity in acute gastroenteritis caused by Norwalk agent. N. Engl. J. Med. 297, 86-89 (1977)

67. Ayukekbong, J. A. et al. Pattern of circulation of norovirus Gll strains during natural infection. J. Clin. Microbiol. 52, 4253-4259 (2014).

68. Simmons, K., Gambhir, M., Leon, J. \& Lopman, B. Duration of immunity to norovirus gastroenteritis. Emerg. Infect. Dis. 19, 1260-1267 (2013).

69. Lindesmith, L. C. et al. Broad blockade antibody responses in human volunteers after immunization with a multivalent norovirus VLP candidate vaccine: immunological analyses from a phase I clinical trial. PLoS Med. 12, e1001807 (2015).

70. Reeck, A. et al. Serological correlate of protection against norovirus-induced gastroenteritis. J. Infect. Dis. 202, 1212-1218 (2010)

71. Ramani, S. et al. Mucosal and cellular immune responses to Norwalk virus. J. Infect. Dis. 212 397-405 (2015)

72. Yi, W. et al. Escherichia coli O86 O-antigen biosynthetic gene cluster and stepwise enzymatic synthesis of human blood group B antigen tetrasaccharide. J. Am. Chem. Soc. 127, 2040-2041 (2005).

73. Rasko, D. A., Wang, G., Monteiro, M. A., Palcic, M. M $\&$ Taylor, D. E. Synthesis of mono- and di-fucosylated type I Lewis blood group antigens by Helicobacter pylori. Eur. J. Biochem. 267 6059-6066 (2000).

74. Ruvoen-Clouet, N. et al. Increase in genogroup II.4 norovirus host spectrum by CagA-positive Helicobacter pylori infection. J. Infect. Dis. $\mathbf{2 1 0}$ 183-191 (2014).

75. Baldridge, M. T. et al. Commensal microbes and interferon- $\lambda$ determine persistence of enteric murine norovirus infection. Science 347, 266-269 (2015). The first demonstration of the role of the bacterial microbiota in the persistence of enteric norovirus infections.

76. Li, L. et al. The fecal viral flora of California sea lions. J. Virol. 85, 9909-9917 (2011).

77. Wu, Z. et al. Deciphering the bat virome catalog to better understand the ecological diversity of bat viruses and the bat origin of emerging infectious diseases. ISME J. 10, 609-620 (2015).

78. Katayama, K. et al. Phylogenetic analysis of the complete genome of 18 Norwalk-like viruses. Virology 299, 225-239 (2002).

79. Siebenga, J. J et al. Phylodynamic reconstruction reveals norovirus GII.4 epidemic expansions and their molecular determinants. PLoS Pathog. 6, e1000884 (2010).

80. Bull, R. A., Eden, J. S., Rawlinson, W. D. \& White, P. A Rapid evolution of pandemic noroviruses of the GII.4 lineage. PLoS Pathog. 6, e1000831 (2010).

81. Eden, J. S. et al. The emergence and evolution of the novel epidemic norovirus Gll.4 variant Sydney 2012 Virology 450-451, 106-113 (2014).

82. Lopman, B. et al. Increase in viral gastroenteritis outbreaks in Europe and epidemic spread of new norovirus variant. Lancet 363, 682-688 (2004).

83. Debbink, K. et al. Emergence of new pandemic Gll.4 Sydney norovirus strain correlates with escape from herd immunity. J. Infect. Dis. 208, 1877-1887 (2013).

84. Lindesmith, L. C. et al. Mechanisms of Gll.4 norovirus persistence in human populations. PLoS Med. 5, e31 (2008).

85. Boon, D. et al. Comparative evolution of GII.3 and GIl.4 norovirus over a 31-year period. J. Virol. 85 , 8656-8666 (2011). 
86. Kobayashi, M. et al. Molecular evolution of the capsid gene in norovirus genogroup I. Sci. Rep. 5, 13806 (2015).

87. Iritani, N. et al. Genetic analysis of the capsid gene of genotype Gll.2 noroviruses. J. Virol. 82, 7336-7345 (2008).

88. Swanstrom, J., Lindesmith, L. C., Donaldson, E. F. Yount, B. \& Baric, R. S. Characterization of blockade antibody responses in Gll.2.1976 Snow Mountain virus-infected subjects. J. Virol. 88, 829-837 (2014).

89. Bull, R. A et al Norovirus recombination in ORF1/ ORF2 overlap. Emerg. Infect. Dis. 11, 1079-1085 (2005).

90. Eden, J. S., Tanaka, M. M., Boni, M. F., Rawlinson, W. D. $\&$ White, P. A. Recombination within the pandemic norovirus GII.4 lineage. J. Virol. 87, 6270-6282 (2013).

91. Mahar, J. E., Bok, K., Green, K. Y. \& Kirkwood, C. D. The importance of intergenic recombination in norovirus GII.3 evolution. J. Virol. 87, 3687-3698 (2013).

92. Chang, K. O., Sosnovtsev, S. V., Belliot, G., King, A. D. \& Green, K. Y. Stable expression of a Norwalk virus RNA replicon in a human hepatoma cell line. Virology 353 , 463-473 (2006).

93. Belliot, G. et al. Norovirus proteinase-polymerase and polymerase are both active forms of RNA-dependent RNA polymerase. J. Virol. 79, 2393-2403 (2005).

94. Subba-Reddy, C. V., Goodfellow, I. \& Kao, C. C. VPgprimed RNA synthesis of norovirus RNA-dependent RNA polymerases by using a novel cell-based assay. J. Virol. 85, 13027-13037 (2011).

95. Subba-Reddy, C. V., Yunus, M. A., Goodfellow, I. G \& Kao, C. C. Norovirus RNA synthesis is modulated by an interaction between the viral RNA-dependent RNA polymerase and the major capsid protein, VP1. J. Virol. 86, 10138-10149 (2012)

96. Gairard-Dory, A. C. et al. Clinical usefulness of oral immunoglobulins in lung transplant recipients with norovirus gastroenteritis: a case series. Transplant. Proc. 46, 3603-3605 (2014).

97. Florescu, D. F., Hill, L. A., McCartan, M. A. \& Grant, W. Two cases of Norwalk virus enteritis following smal bowel transplantation treated with oral human serum immunoglobulin. Pediatr. Transplant. 12, 372-375 (2008).

98. Chachu, K. A. et al. Antibody is critical for the clearance of murine norovirus infection. J. Virol. 82, 6610-6617 (2008).

99. Green, K. Y. Norovirus infection in immunocompromised hosts. Clin. Microbiol. Infect. 20, 717-723 (2014).
100. Rossignol, J. F. \& El-Gohary, Y. M. Nitazoxanide in the treatment of viral gastroenteritis: a randomized doubleblind placebo-controlled clinical trial. Aliment. Pharmacol. Ther. 24, 1423-1430 (2006).

101. Siddiq, D. M., Koo, H. L., Adachi, J. A. \& Viola, G. M. Norovirus gastroenteritis successfully treated with nitazoxanide. J. Infect. 63, 394-397 (2011).

102. Woodward, J. M. et al. The role of chronic norovirus infection in the enteropathy associated with common variable immunodeficiency. Am. J. Gastroenterol. 110, 320-327 (2015).

103. Nice, T. J. et al. Interferon- $\lambda$ cures persistent murine norovirus infection in the absence of adaptive immunity. Science 347, 269-273 (2015).

104. Chang, K. O. \& George, D. W. Interferons and ribavirin effectively inhibit Norwalk virus replication in repliconbearing cells. J. Virol. 81, 12111-12118 (2007).

105. Richardson, C., Bargatze, R. F., Goodwin, R. \& Mendelman, P. M. Norovirus virus-like particle vaccines for the prevention of acute gastroenteritis. Expert Rev. Vaccines 12, 155-167 (2013).

106. Bernstein, D. I. et al. Norovirus vaccine against experimental human GII. 4 virus illness: a challenge study in healthy adults. J. Infect. Dis. 211, 870-878 (2015).

107. Kocher, J. et al. Intranasal P particle vaccine provided partial cross-variant protection against human GII.4 norovirus diarrhea in gnotobiotic pigs. J. Virol. 88, 9728-9743 (2014)

108. Tamminen, K., Lappalainen, S., Huhti, L., Vesikari, T. \& Blazevic, V. Trivalent combination vaccine induces broad heterologous immune responses to norovirus and rotavirus in mice. PLOS ONE 8, e70409 (2013).

109. van der Vries, E. et al. Prolonged influenza virus shedding and emergence of antiviral resistance in immunocompromised patients and ferrets. PLOS Pathog. 9, e1003343 (2013).

110. Huynen, P. et al. Molecular epidemiology of norovirus infections in symptomatic and asymptomatic children from Bobo Dioulasso, Burkina Faso. J. Clin. Virol. 58 515-521 (2013).

111. Hickman, D. et al. The effect of malnutrition on norovirus infection. mBio 5, e01032-13 (2014).

112. Frange, P. et al. Prevalence and clinical impact of norovirus fecal shedding in children with inherited immune deficiencies. J. Infect. Dis. 206, 1269-1274 (2012)

113. Meeroff, J. C., Schreiber, D. S., Trier, J. S. \& Blacklow, N. R. Abnormal gastric motor function in viral gastroenteritis. Ann. Intern. Med. 92, 370-373 (1980).

114. Schwartz, S. et al. Norovirus gastroenteritis causes severe and lethal complications after chemotherapy and hematopoietic stem cell transplantation. Blood 117, 5850-5856 (2011)

115. Schreiber, D. S., Blacklow, N. R. \& Trier, J. S. The small intestinal lesion induced by Hawaii agent acute infectious nonbacterial gastroenteritis. J. Infect. Dis. 129, 705-708 (1974).

116. Troeger, H. et al. Structural and functional changes of the duodenum in human norovirus infection. Gut $\mathbf{5 8}$, 1070-1077 (2009).

117. Desai, R. et al. Severe outcomes are associated with genogroup 2 genotype 4 norovirus outbreaks: a systematic literature review. Clin. Infect. Dis. $\mathbf{5 5}$ 189-193 (2012).

118. Huhti, L. et al. Norovirus GIl-4 causes a more severe gastroenteritis than other noroviruses in young children. J. Infect. Dis. 203, 1442-1444 (2011).

119. Chan, M. C. et al. Fecal viral load and norovirusassociated gastroenteritis. Emerg. Infect. Dis. 12, 1278-1280 (2006).

120. Kroneman, A. et al. Proposal for a unified norovirus nomenclature and genotyping. Arch. Virol. 158 2059-2068 (2013).

Acknowledgements

This work was supported by the European Union's Horizon 2020 grant to the COMPARE Consortium, under grant agreement number 643476, and by the Virgo Consortium, funded by the Dutch Government (project number FES0908). The authors thank J. Le Pendu for helpful comments.

Competing interests statement

The authors declare no competing interests.

DATABASES

RCSB Protein Data Bank:

http://www.rcsb.org/pdb/home/home.do

4WZ

FURTHER INFORMATION

Norovirus Genotyping Tool:

http://www.rivm.nl/mpf/norovirus/typingtool

ALL LINKS ARE ACTIVE IN THE ONLINE PDF 